\title{
RELIABILITY OF THE WASSERMANN REACTION
}

neoarsphenamine in addition to the local treatment. A mumber of cases had been treated for syphilis and trichomonas concurrently, but she did not think there was much effect on the trichomonas by the use of the intravenous arsenic.

\section{ANNOTATION}

\section{RELIABILITY OF THE WASSERMANN REACTION}

In the present issue will be found a paper on the use and significance of serum tests for syphilis by Colonel L. W. Harrison and Brigadier T. E. Osmond. The authors were at some pains to point out the various conditions, both physiological and pathological, which may give rise to false positive results and it has been suggested that this might conceivably engender, in the minds of medical men, a feeling of "Wassermann insecurity." In reality-and a careful consideration of the paper will confirm this-nothing could be further from the truth, and a Wassermann reaction carried out by a competent pathologist is amongst the most reliable of pathological tests which we have at our command. The Wassermann reaction, and particularly the method in question (No. 1 method M.R.C.) has proved itself throughout a number of searching enquiries to be highly specific and reasonably sensitive.

The point which we should like to stress is that it is not the test which is mainly at fault but its interpretation. In the past there has been far too little critical judgment used in assessing the results of Wassermann reactions and even today many practitioners are inclined to say " positive W.R., therefore syphilis." Whilst it is admitted that if we exclude certain tropical and sub-tropical diseases such as yaws, leprosy and malaria there are few conditions which are likely to give a persistently false positive W.R., nevertheless experience has shown that the danger does exist. Not only certain diseases, but some ill-understood physiological conditions do from time to time give positive results with serum tests for syphilis, and the clinician will do well to bear this in mind. The pathologist is not a diagnostician ; it is his job to report the results which he gets, not to assess them, though in difficult cases and where unexpected results are obtained a consultation with him will often prove useful. The Wassermann test, like all other tests, has its limitations ; and when the clinician is faced with the assessment of a result he will do well to bear this in mind. It is when a positive reaction is reported in a case in which there is nothing in the history or clinical signs pointing to syphilis that the greatest difficulty lies. It is no light thing to diagnose syphilis and thereby condemn the patient to the long and somewhat dangerous treatment which such a diagnosis entails. When such a case does occur the clinician should remember the limitations of serum tests and leave no stone unturned to try to discover why the result was positive; he will then go over in his mind the various causes of false positive reactions and seek to exclude them before making a diagnosis of syphilis.

Another further point perhaps needs stressing; most of the conditions met with in this country which are liable to give a fałse positive Wassermann reaction only do so over comparatively short periods, and if repeated tests are carried out over a sufficiently long time the true nature of the condition will usually be revealed. The Wassermann test, particularly that as modified by Richardson, and the Kahn, including the Verification test, are amongst the most reliable tests we have at our disposal if assessed with judgment but they are not 100 per cent perfect. If the clinician will bear this in mind and remember that a positive serum test does not in itself justify a diagnosis of syphilis he will not be likely to go far wrong. The specificity and sensitivity of the Wassermann reactions are probably greater today than at any time in the past, thanks to the work carried out by serologists all over the world; the only proviso is that a reliable technique should be employed and the test carried out by a pathologist of experience. 